\title{
Absolute height estimation using a single TerraSAR-X Staring Spotlight acquisition
}

\author{
Sergi Duque, Helko Breit, Ulrich Balss and Alessandro Parizzi
}

\begin{abstract}
The work presented in this letter exploits the long Synthetic Aperture Radar (SAR) of a single TerraSAR-X Staring Spotlight (ST) acquisition to derive absolute heights. Here, the slight azimuth defocussing effect due to height mismatch between true height and the height assumed in SAR focusing is analyzed. The impact is almost negligible for most of acquisition modes. In contrast, spaceborne modes with very long aperture, such as TerraSAR-X ST acquisition mode, present sensibility that can be used to retrieve absolute heights. The accuracy depends on incidence angle, orbit type and mainly on Signal to Clutter Ratio (SCR). Two different results are presented to demonstrate that absolute heights can be retrieved with an accuracy of few meters using a single TerraSAR-X ST acquisition.
\end{abstract}

Index Terms-TerraSAR-X, Staring Spotlight, absolute height.

\section{INTRODUCTION}

$\mathbf{T}$ HE Synthetic Aperture Radar (SAR) has demonstrated to be an useful instrument for earth observation in the last decades. Several acquisition modes have been tested and many processing algorithms have been developed. The main modes are Stripmap (SM), ScanSAR, TOPS and Spotlight. In general, there is a trade-off between the size of the imaged area and the achieved resolution. The Staring Spotlight (ST) mode aims for a better azimuth resolution despite imaging a smaller area. This new TerraSAR-X mode consists on steering the antenna to a fixed point on ground during the whole acquisition time. The azimuth steering angles range from $-2.2^{\circ}$ to $2.2^{\circ}$. The associated azimuth bandwidth is around $38 \mathrm{KHz}$ yielding an azimuth resolution below $24 \mathrm{~cm}$ [1]. Some assumptions of TerraSAR-X Multi-Mode SAR Processor (TMSP) had to be reviewed in order to properly implement the ST mode and to achieve its very high azimuth resolution. Effects like the tropospheric delay, orbit curvature and higher orders in the range history had to be taken into account [1]. A small mismatch on the azimuth focusing has a bigger impact on a Staring Spotlight acquisition than on a Stripmap due to the larger integration time. A relative small height offset assumed during focusing may derive in a slight resolution loss. This letter demonstrates that, in general, the defocus due to this effect is insignificant for imaging purposes. However, it can be analyzed for long apertures and the absolute height can be derived within few meters accuracy under certain Signal to Clutter Ratio (SCR) conditions. This method can be considered an alternative to other methods intended to obtain absolute 3D positioning from SAR acquisitions. Other techniques like

The authors are with the Remote Sensing Technology Institute (IMF), German Aerospace Center (DLR), 82234 Oberpfaffenhofen, Germany (email: sergi.duquebiarge@dlr.de; helko.breit@dlr.de; ulrich.balss@dlr.de and alessandro.parizzi@dlr.de)
Radargrametry [2] and Multi-Chromatic Analysis (MCA) [3] retrieve absolute positions of point targets with accuracies typically on the meter or sub-meter level but they need at least two SAR acquisitions. The proposed method in this letter only needs a single long aperture acquisition at the price of a worsened accuracy.

The letter is structured as follows. The azimuth focusing impact of height offset, tropospheric delay and orbit inaccuracies are depicted in Section II. Section III presents the processing flow and the derived estimation accuracy. First results over two different scenarios are illustrated in Section IV. Finally, a brief summary and conclusions are drawn is Section V.

\section{IMPACT OF HEIGHT OFFSET IN TERRASAR-X AZIMUTH FOCUSING}

The well known azimuth Frequency Modulation (FM) rate for a target is typically described as

$$
F M_{\text {rate }}=\frac{2 B}{\lambda R_{0}}
$$

where $\lambda$ is the wavelength, $R_{0}$ is the range at closest approach and $B$ is the velocity parameter. The term $R_{0}$ varies considerably depending on the incidence angle. For a fixed platform height, steeper incidence angles yield in shorter ranges and consequently in higher FM rates. If a second Taylor order is assumed for range derivation, $B$ can be expressed as $B=\dot{\vec{R}} \cdot \dot{\vec{R}}-\vec{R} \cdot \ddot{\vec{R}}$, being $\vec{R}, \dot{\vec{R}}$ and $\ddot{\vec{R}}$ the range, velocity and acceleration vector respectively. Then, in the classical SAR approach where the sensor moves with constant velocity in a rectilinear trajectory parallel to the scene plane, there is no sensitivity to target height variation. In the orbital case, height sensitivity is due to the non zero term $\vec{R} \cdot \ddot{\vec{R}}$. The range vector depends on the target height and the curved orbits yield in a significant acceleration vector. In this case, the estimated azimuth FM rate slightly depends on terrain height. Therefore, it may exist a small FM rate mismatch in azimuth compression. In order to give an expression that links the FM rate mismatch $(\Delta F M)$ and the height offset $(\Delta h)$ let us assume a locally flat orbit with a satellite velocity $\left(V_{\text {sat }}\right)$ and acceleration related to the gravity at satellite's height $\left(g_{H s}\right)$. If the Earth curvature is not taken into account, the velocity parameter can be approximated as

$$
B \approx V_{\text {sat }}^{2}-R_{0} \cdot g_{H s} \cdot \cos \left(\theta_{\text {inc }}\right),
$$

being $\theta_{\text {inc }}$ the incidence angle. The FM rate mismatch can be approximated as

$$
\Delta F M \approx \frac{2 g_{H s}}{\lambda R_{0}} \cdot \Delta h
$$


In order to validate this approximation, the FM rate mismatch has been numerically calculated for a full 90 minutes TerraSAR-X reference orbit. The FM rate has been obtained for all latitudes and for three different incidence angles assuming the ellipsoidal Earth model WGS-84 and a fixed reference height of $700 \mathrm{~m}$ above the ellipsoid. Then, the FM rate calculation has been repeated varying the height 25, 50 and $100 \mathrm{~m}$. The differences with the first calculation yield in the corresponding FM rate mismatches. According to TerraSAR-X reference orbit, the FM rate mismatch is illustrated in Figure 1 for a set of incidence angles and height offsets of 25 (dotted line), 50 (dashed line) and $100 \mathrm{~m}$ (solid line). Here, latitudes $0^{\circ}$ and $180^{\circ}$ refer satellite pass by South and North pole while latitudes $90^{\circ}$ and $270^{\circ}$ refer pass by equator in ascending and descending respectively. The plots in black are related to an incidence angle of $21^{\circ}$ while the plots in blue and red refer to incidence angles of $47.3^{\circ}$ and $58^{\circ}$ respectively. Notice that steeper incidence angles show greater FM rate mismatch. It has to be highlighted that earth rotation has also an impact. In a right looking spaceborne sensor descending orbits present higher sensitivity than ascending. The FM rate mismatch for a height offset of $100 \mathrm{~m}$ is derived for the three incidence angles using Equation (3). The approximated values are represented with a thin solid flat line in black for $\theta_{i n c}=21^{\circ}$, in blue for $\theta_{i n c}=47^{\circ}$ and in red for $\theta_{i n c}=58^{\circ}$.

The impulse response at the output of the matched filter in azimuth frequency domain is given by [4]:

$$
S\left(f_{a z}\right)=\operatorname{rect}\left(\frac{f_{a z}}{B w_{a z}}\right) \cdot e^{-j \pi \frac{\Delta F M_{\text {rate }} f_{a z}^{2}}{F M_{\text {rate }}^{2}}} \cdot e^{-j 2 \pi f_{a z} t_{a z}},
$$

where $f_{a z}$ is the azimuth frequency, $B w_{a z}$ is the total azimuth bandwidth and $t_{a z}$ is the zero Doppler azimuth time. The defocus is introduced by the first exponential term, which is dependent on the FM rate, FM rate mismatch and the azimuth bandwidth. Since, it is inversely dependent on the FM rate square and just linearly with the FM rate mismatch, acquisitions with flatter angles are more sensitive to present defocus. Therefore, they will present also better accuracies when retrieving absolute heights. In order to show the defocus effect introduced by a height offset in a SM and ST acquisitions, the azimuth impulse response broadening has been simulated for the selected incidence angles. The FM rate and the mismatches correspond to the numerically calculated values at $48.08^{\circ} \mathrm{N}$ latitude corresponding to DLR in Oberpfaffenhofen, Germany. The azimuth bandwidth for SM mode is set to $2.8 \mathrm{~K} \mathrm{~Hz}$ and $38.3 \mathrm{KHz}$ for ST. The broadening has been calculated taking

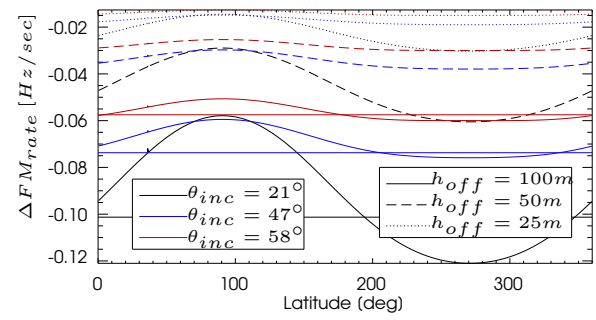

Fig. 1: FM rate mismatch for different incidence angles and height offsets over a complete TerraSAR-X reference orbit. into account the azimuth Hamming window used in TerraSAR$\mathrm{X}$ azimuth processing and calculated w.r.t. a zero FM rate mismatch. It is defined as percentage by

$$
\operatorname{Broad}(\%)=\left(\frac{\Delta t_{a z, \text { defocus }}}{\Delta t_{a z, \text { nom }}}-1\right) \times 100 \%
$$

where $\Delta t_{a z, \text { defocus }}$ is the azimuth peak wide in time when there is a defocus effect and $\Delta t_{a z, n o m}$ when it is perfectly focused. Table \ summarizes the broadening in SM and ST for heights offsets of 50 and $100 \mathrm{~m}$ and for previous mentioned incidence angles. It can be seen that a height offset in

\begin{tabular}{|c||c|c||c|c|}
\hline \multirow{2}{*||}{$\theta_{\text {inc }}$} & \multicolumn{2}{c||}{ ST Broadening } & \multicolumn{2}{c|}{ SM Broadening } \\
\cline { 2 - 5 } & $\Delta h=50 \mathrm{~m}$ & $\Delta h=100 \mathrm{~m}$ & $\Delta h=50 \mathrm{~m}$ & $\Delta h=100 \mathrm{~m}$ \\
\hline \hline $21^{\circ}$ & $4.7 \%$ & $14.7 \%$ & $7 \mathrm{e}-5 \%$ & $18 \mathrm{e}-5 \%$ \\
$47^{\circ}$ & $9.9 \%$ & $59.9 \%$ & $18 \mathrm{e}-5 \%$ & $74 \mathrm{e}-5 \%$ \\
$58^{\circ}$ & $15.7 \%$ & $116.0 \%$ & $30 \mathrm{e}-5 \%$ & $119 \mathrm{e}-5 \%$ \\
\hline
\end{tabular}

TABLE I: Azimuth impulse response broadening for a SM and ST acquisition with different incidence angles and height offsets.

azimuth focusing produces practically no defocussing in a SM acquisition. Also, in a ST acquisition whit height offsets below $50 \mathrm{~m}$ the azimuth impulse response is worsened less than $10 \%$ in the major part of the cases. The effect starts to be appreciable in the ST amplitude image when the acquisition is performed with a flat incidence angle and the height offset is above $100 \mathrm{~m}$.

\section{A. Tropospheric path delay impact}

The tropospheric delay has to be accounted for in a spaceborne long synthetic aperture acquisition modes [1]. The TMSP for ST processing assumes a fix Zenith Path Delay (ZPD) at sea level of $2.3 \mathrm{~m}$ which corresponds to an approximation of the dry part of the tropospheric delay. The ZPD at sea level is scaled to scene height [5]. Then, it is projected into slant range for all the aperture integrated in the azimuth compression. Small discrepancies regarding the wet part which depends on atmospheric conditions at acquisition time can be neglected and do not significantly affect the focused image quality. However, the effect introduced by the tropospheric delay is similar to height offset effect. Therefore, small unaccounted wet delays have the same effect than an added height offset. According to [6], the wet part component in the ZPD varies in the order of few set of ten centimeters. Figure 2 represents the FM rate mismatch for a $48.08^{\circ} \mathrm{N}$ latitude corresponding to Oberpfaffenhofen, Germany, in ascending orbit. The FM rate mismatch is numerically calculated for the three incidence angles and also assuming three different tropospheric delays for every incidence angle. In Figure 2 the true tropospheric delay has been set to $2.7 \mathrm{~m}$ in all cases, the FM rate mismatch when assuming the correct delay is plotted in dashed lines $\left(\varepsilon_{\text {tropo }}=0 \mathrm{~cm}\right)$, the dotted and solid lines correspond to processing with a delay error of 20 and $40 \mathrm{~cm}$ respectively $\left(\varepsilon_{\text {tropo }}=20 \mathrm{~cm}\right.$ and $\left.\varepsilon_{\text {tropo }}=40 \mathrm{~cm}\right)$. As it can be seen, the mismatch presents a linear behavior w.r.t. height 


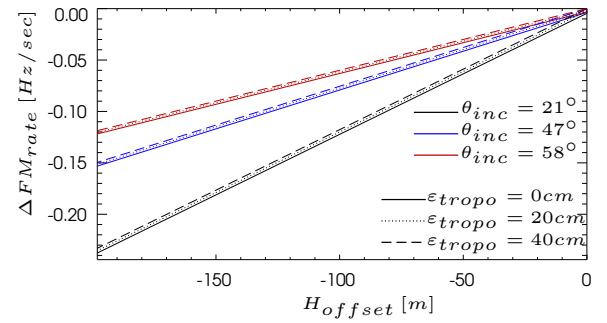

Fig. 2: FM rate mismatch for the selected incidence angles with a real tropospheric delay of $2.7 \mathrm{~m}$. Tropospheric error delay in processing of $0 \mathrm{~cm}$ (dashed lines), $20 \mathrm{~cm}$ (dotted lines) and $40 \mathrm{~cm}$ (solid lines).

offset with different slope depending on the incidence angle. The tropospheric delay discrepancy introduces an offset of the FM rate mismatch which is equivalent to an equivalent height bias, $h_{e q}$, of few meters. Table $\amalg$ shows this equivalent height bias assuming 20 and $40 \mathrm{~cm}$ tropospheric delay errors when the true tropospheric delay is $2.7 \mathrm{~m}$.

\begin{tabular}{|c||c|c|}
\hline$\theta_{i n c}$ & $h_{e q}$ for $\varepsilon_{\text {tropo }}=20 \mathrm{~cm}$ & $h_{e q}$ for $\varepsilon_{\text {tropo }}=40 \mathrm{~cm}$ \\
\hline $21^{\circ}$ & $1.9 \mathrm{~m}$ & $3.9 \mathrm{~m}$ \\
\hline $47.3^{\circ}$ & $2.4 \mathrm{~m}$ & $4.8 \mathrm{~m}$ \\
\hline $58^{\circ}$ & $2.6 \mathrm{~m}$ & $5.2 \mathrm{~m}$ \\
\hline
\end{tabular}

TABLE II: Equivalent height bias for different tropospheric delays errors in focusing and incidence angles when the real tropospheric delay is $2.7 \mathrm{~m}$.

\section{B. Orbit inaccuracies impact}

The orbit inaccuracies have also an impact on the FM rate. Therefore, when the absolute height is derived it contains a bias due to not having a perfect orbit. It is possible to get the FM rate sensitivity w.r.t. range orbit inaccuracy taking into account the approximation given by (2). This is

$$
\Delta F M_{o r b} \approx-\frac{2 V_{s a t}^{2}}{\lambda R_{0}^{2}} \cdot \Delta R_{o r b} .
$$

When this FM rate mismatch is assumed to be due to height offset, the bias in the height offset estimation is

$$
\Delta h_{\text {orb }, \text { bias }}=-\frac{V_{\text {sat }}^{2}}{g_{H s} R_{0}} \cdot \Delta R_{\text {orb }} .
$$

In the case of TerraSAR-X, orbits are really accurate. In [7] it is demonstrated TerraSAR-X orbit accuracy in range direction is below $2 \mathrm{~cm}$. Then, assuming $\sigma_{R_{\text {orb }}}=2 \mathrm{~cm}$, $V_{\text {sat }}=7600 \mathrm{Km} / \mathrm{h}, H_{s}=514 \mathrm{Km}$ and $R_{0}=754 \mathrm{Km}$ the standard deviation of the height bias is just $18 \mathrm{~cm}$. The orbit inaccuracies impact in the case of using TerraSAR-X acquisitions is relatively small, just a height estimation bias of few centimeters is introduced. In case of applying the proposed method to other sensors, the impact of orbit inaccuracies should be analyzed.

\section{Processing Flow AND estimation acCuracy}

The proposed method to analyze the ST image defocus and retrieve absolute heights is based on estimating the azimuth position variation of point targets within different azimuth sub-apertures. Taking into account that long apertures are not fully characterized by linear FM rates, the azimuth position displacement of a point target due to a FM rate mismatch in the n-th sub-aperture can be expressed as [4]

$$
\Delta A z_{n}=\frac{\Delta F M_{\text {rate }, n}}{F M_{\text {rate }, n}^{2}} \cdot f_{a z, n} \cdot V_{\text {sat }, g r},
$$

being $\Delta F M_{\text {rate }, n}$ and $F M_{\text {rate }, n}$ the FM rate mismatch and FM rate corresponding to the n-th sub-aperture, $V_{s a t, g r}$ the satellite velocity on ground and $f_{a z, n}$ the sub-aperture central frequency. The proposed algorithm starts with getting the ST input image ready for sub-aperture processing. A deramping operation according to the Doppler centroid of each azimuth time is applied to the image. Then, azimuth spectral weighting is removed to give the same relevance to all sub-bands. The next step is to generate the independent azimuth sub-bands and to reramp them according to previous deramping. The point targets candidates are selected by setting a sub-band SCR threshold. Thus, the selected point targets are those ones that show a small SCR variation within sub-bands (a margin of $\pm 2 d B$ has been used) assuming certain azimuth displacement and with no range position variation. Finally, the azimuth position variation is linked to an absolute height. In order to establish this link, $\Delta F M_{\text {rate }, n}$ and $F M_{\text {rate }, n}$ are calculated numerically for each sub-band and for a certain height. The expected azimuth displacements due to the selected height are derived using (8). Therefore, it is possible to perform a search in height domain to find the height that gives the least root mean square of the difference between the measured and the expected. A priori info is not strictly necessary, the height search can be performed in two steps. First over a very large dynamic range using a coarse grid and getting a rough height result. Then, over a smaller range around the obtained coarse height with a finner grid. If the range dynamic can be coarsely a priori known, the computation cost can be significantly reduced. The a priori information can be just a coarse estimation of the scene height variation, minimum and maximum height. Let us assume now a linear FM rate in order to derive an expression for height accuracy. The azimuth subband time shift can be expressed by

$$
\Delta t_{a z}[n]=\beta \cdot f_{a z}[n] \quad n=0, \ldots, N_{s u b}-1,
$$

where $f_{a z}[n]$ is the azimuth center frequency of the n-th subband, $N_{s u b}$ is the number of sub-bands and $\beta$ is the constant defined as $\beta=\frac{\Delta F M_{\text {rate }}}{F M_{\text {rate }}^{2}}$. The azimuth frequency is given by $\Delta f_{a z}[n]=-\frac{B w_{a z}}{2}+\frac{B w_{a z}}{2 N_{s u b}}+\frac{B w_{a z}}{N_{s u b}} \cdot n \quad n=0, \ldots, N_{s u b}-1$,

According to $[8], \beta$ can be estimated with the following accuracy

$$
\sigma_{\beta}=\frac{\sigma_{\Delta t_{a z}}}{\sqrt{\sum_{n=0}^{N_{s u b}-1}\left(f_{a z}[n]\right)^{2}}} .
$$

The azimuth time positioning accuracy for each sub-band is given by [9]

$$
\sigma_{\Delta t_{a z}}=\sqrt{\frac{3}{2}} \cdot \frac{N_{s u b} \sqrt{N_{s u b}}}{\pi B w_{a z} \sqrt{S C R}}
$$




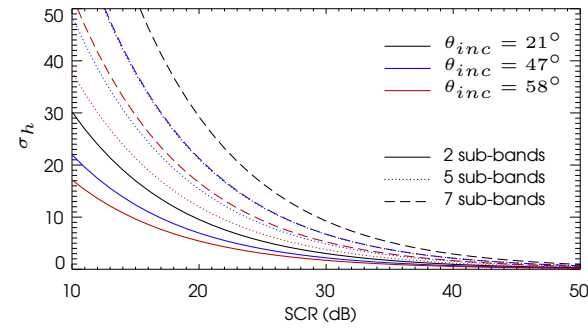

Fig. 3: Theoretical height accuracy depending on SCR for different incidence angles and number of sub-bands.

In order to give a compact expression for the obtained height accuracy, $R_{0}$ can be substituted by $\frac{H_{s}}{\cos \left(\theta_{\text {inc }}\right)}$. Thus, taking into account the gravity at satellite's height and putting all the equations together, the height accuracy is given by

$$
\begin{aligned}
\sigma_{h}= & \frac{2 \cdot V_{s a t}^{4} \cdot \cos \left(\theta_{i n c}\right) \cdot\left(R_{\text {Earth }}+H_{s}\right)^{2}}{M \cdot G \cdot \lambda \cdot H_{s} \cdot \pi \cdot B w_{a z}^{2}} . \\
& \cdot \sqrt{\frac{18 N_{\text {sub }}^{4}}{S C R \cdot\left(N_{\text {sub }}^{2}-1\right)}}
\end{aligned}
$$

Here, $G$ is the gravitational constant $\left(6.673 \times 10^{-11} N\right.$. $\left.(\mathrm{m} / \mathrm{kg})^{2}\right), M$ is the total Earth mass $\left(\approx 5.97 \cdot 10^{24} \mathrm{~kg}\right)$ and $R_{\text {Earth }}$ is the Earth radius. Notice that the obtained expression mainly depends on geometric parameters, such as incidence angle and satellite height. Even the azimuth bandwidth could be expressed as function of squint angles. This expression shows how the accuracy is improved quadratically w.r.t. azimuth bandwidth and the better performance of flat incidence angles. Regarding the number of subapertures, the height accuracy is proportionally worsened with $N_{s u b}$. Figure 3 shows the expected height accuracy for the three selected incidence angles and for 2, 5 and 7 sub-bands assuming $H_{s}=514 \mathrm{Km}$ and $V_{\text {sat }}=7600 \mathrm{Km} / \mathrm{h}$. The theoretical accuracy is improved when less sub-bands are used. However, in practice, more than two sub-bands are needed to discriminate what really behaves like a point target with a practically linear FM rate mismatch. In addition, there may be other source of errors like an uncertainty of the central frequency due to non perfect unweightening. In that case, the azimuth time positioning accuracy would be given by

$$
\sigma_{t_{a z}}^{\prime}=\sqrt{\sigma_{t_{a z}}^{2}+\beta^{2} \cdot \sigma_{f_{N c}}^{2}},
$$

being $\sigma_{f_{N c}}$ the uncertainty of the central frequency for each sub-band. This kind of effect is mitigated when more than two sub-bands are used. Therefore, a priori a reduced number of sub-bands, 3 to 5 , would give a better performance.

\section{REsults}

This section presents two different cases where the absolute height has been retrieved using a single ST acquisition.

\section{A. Corner Reflector scenario}

The first scenario is an ST ascending acquisition over DLR in Oberpfaffenhofen, Germany. Nine corner reflectors with edges $1.5 \mathrm{~m}$ long have been deployed among an airfield. The corners present a SCR of $50 \mathrm{~dB}$. The ST acquisition was performed with an incidence angle of $47.3^{\circ}$ and azimuth bandwidth of $38.3 \mathrm{~K} \mathrm{~Hz}$ corresponding to a nominal resolution of $23 \mathrm{~cm}$. The point target azimuth displacement has been carried out using different number of non overlapped subbands. A TanDEM-X Intermediate DEM (IDEM) has been used as a reference to validate the obtained heights [10]. The IDEM is calibrated and the height accuracy for region of interest is $70 \mathrm{~cm}$. First, the height derivation was carried out setting the number of sub-bands to 5 and assuming the tropospheric delay used in processing. The difference between the obtained heights and the IDEM taking into account the 9 CRs presented a bias of $3.6 \mathrm{~m}$ with a standard deviation of $1.2 \mathrm{~m}$. Notice that taking into account the IDEM accuracy, these $\sigma_{h-I D E M}=1.2 \mathrm{~m}$ yield in a retrieved height accuracy of $\sigma_{h}=97 \mathrm{~cm}$. Then, the tropospheric delay was calculated for the region of interest at the acquisition time using the 3-D numerical weather model data from the European Center for Medium-Range Weather Forecast (ECMWF) [6]. The obtained equivalent delay at sea level is $2.53 \mathrm{~m}$ instead the $2.3 \mathrm{~m}$ assumed in the standard ST processing. The tropospheric delay discrepancy was taken into account for the height calculation. As consequence, the obtained bias was just $0.8 \mathrm{~m}$, which most probably corresponds mainly to CRs phase center w.r.t. ground and there may be also a small contribution due to orbit inaccuracy effect. The tropospheric delay just modifies the bias while standard deviation remains $1.2 \mathrm{~m}$. The processing has been carried out also for 2, 3, 7, 12 and 15 sub-bands. The standard deviation of the discrepancy between the obtained heights and IDEM is plotted in Figure 4 Here, the black line represents the theoretical values according to (13) and IDEM accuracy, the blue line assumes an uncertainty of $100 \mathrm{~Hz}$ for sub-bands central frequency. The measured height minus the corresponding IDEM are plotted in turquoise triangles. Since there are only nine measurements to estimate the obtained accuracy, it is necessary to plot the error bars of the accuracy estimation. The error bars are plotted for three times the standard deviation. Notice that the measurements agree with the expected theoretical values. There is also significantly accuracy worsening when the number of sub-bands is increased. However, the minimum number of two sub-bands do not show the best performance. This effect is most probably due to a the small uncertainty of the central frequency for each sub-band. This effect is minimized for a greater number of sub-bands, it is seen on the graph as the two lines converge for higher $N_{\text {sub }}$ values. So, in practice, the optimum number of sub-bands is around $3-5$. These result demonstrate that for point targets with high SCR is possible to obtain their absolute height using a single ST acquisition with accuracies on the meter level.

\section{B. Rio - Antirrio bridge scenario}

The second scenario corresponds to the Rio - Antirrio bridge in Greece. Natural point targets are identified in the nearby of the pylons. Figure 5 shows and optical image of the bridge with a zoom of the area of interest. The highest point of the bridge span is at $86 \mathrm{~m}$ above the reference ellipsoid WGS-84. The amplitude SAR image is overlayed on the 


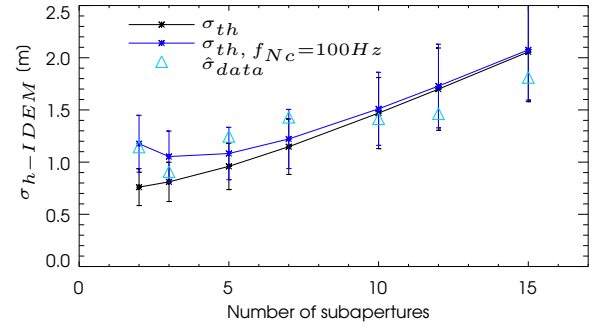

Fig. 4: Accuracy of derived heights with IDEM as reference.

zoom over GoogleEarth with a 3-D model of the bridge. The selected point targets processed using $N_{s u b}=5$ are marked in turquoise diamonds. It is possible to see that the selected points are aligned forming two parallel lines. They correspond mainly to the light poles and few to the crash barrier nearby, both effects are visible on the 3-D model. The points are located not further than 200 meters along the bridge. Both scattering mechanism, pole and barrier, are related to a double bounce with phase centers located on the ground. Thus, it is possible to assume that their phase centers are located at the same height. Since all the points are at the same height and nearby, the tropospheric delay can also considered the same. In this case, the precise tropospheric delay was not available and the fixed dry delay of $2.3 \mathrm{~m}$ was employed. However, here the height accuracy is much bigger than the tropospheric delay discrepancy effect. The ST acquisition has

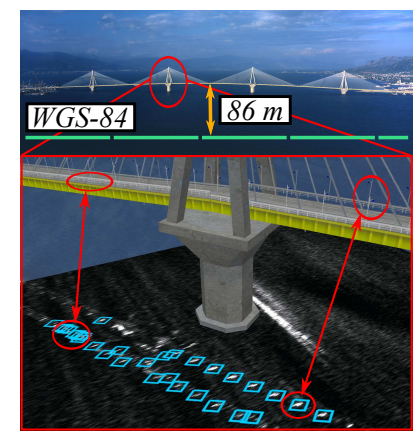

Fig. 5: Optical Rio - Antirrio bridge image.

been performed in a descending orbit with an incidence angle of $26.3^{\circ}$. The proposed algorithm has been done for $2,3,5,7$ and 10 sub-bands. The full band SCR threshold is set to $15 \mathrm{~dB}$. The number of selected points varies between 35 and 30 for 2, 3 and 5 sub-bands. However, for 7 and 10, the selected points are 17 and 7 respectively. This is due to noisy subbands positioning measurements when increase the number of sub-bands. Table [II shows obtained height means and standard deviations. The statistics are derived by using the height values

\begin{tabular}{|c||c|c|c|c|c|}
\hline$N_{s u b}=$ & 2 & 3 & 5 & 7 & 10 \\
\hline \hline$\mu_{h}[\mathrm{~m}]$ & 78.9 & 85.7 & 85.5 & 95.5 & 41.7 \\
\hline$\sigma_{h}[\mathrm{~m}]$ & 17.2 & 13.4 & 12.0 & 36.2 & 277.2 \\
\hline
\end{tabular}

TABLE III: Statistical analysis for the obtained heights.

obtained for all selected point targets at each corresponding sub-band processing. Two sub-bands are not optimal to detect point targets with a defocus due to height offset. Three and five sub-bands show the best performance. The derived heights using 3 and 5 sub-bands match the expected bridge height at the span level. This result shows that it is possible to apply this technique using natural point targets and retrieve heights with accuracies of few tens of meters.

\section{SUMmARY AND CONCLUSIONS}

This work shows the potential of spaceborne long aperture SAR acquisitions. Their geometries with long curved apertures can be exploited to analyze the azimuth defocussing due to a height offset assumed in focusing. This paper analyses this defocussing effect for point targets and proposes a method based on sub-apertures to obtain their absolute height. The authors have derived a general expression for height accuracy. In addition, the impact of relevant effects like tropospheric delay, orbit inaccuracies and Doppler centroid estimation have been discussed and analyzed. Two practical experiments have been shown. The first one over a controlled scenario with a set of nine corners with a very high SCR. The obtained heights have been compared with a TanDEM-X IDEM. The results show an accuracy near the meter level with a good agreement w.r.t. expected accuracy from theory. The second experiment shows that it is possible to achieve a height accuracy of few tens of meters for natural targets over a more realistic scenario. In practice, it has been observed that the optimum number of sub-apertures to be used ranges from 3 to 5 . It has to be highlighted that this technique obtains absolute heights with no need of any reference. Thus, it could be used in several interferometric applications as an absolute reference.

\section{REFERENCES}

[1] Prats-Iraola et al. On the Processing of Very High Resolution Spaceborne SAR Data. Geoscience and Remote Sensing, IEEE Transactions on, 52(10):6003-6016, Oct 2014.

[2] F Leberl. Radargrammetric Image Processing. Artech House, Norwood, Mass., 1989.

[3] F. Bovenga, V.M. Giacovazzo, A. Refice, and N. Veneziani. Multichromatic Analysis of InSAR Data. Geoscience and Remote Sensing, IEEE Transactions on, 51(9):4790-4799, Sept 2013.

[4] I.G. Cumming and F.H. Wong. Digital Processing of Synthetic Aperture Radar Data: Algorithms and Implementation. Artech House remote sensing library. Artech House, 2005.

[5] M. Eineder, C. Minet, P. Steigenberger, Xiaoying Cong, and T. Fritz. Imaging Geodesy - Toward Centimeter-Level Ranging Accuracy With TerraSAR-X. Geoscience and Remote Sensing, IEEE Transactions on, 49(2):661-671, Feb 2011.

[6] Xiaoying Cong, U. Balss, M. Eineder, and T. Fritz. Imaging Geodesy - Centimeter-Level Ranging Accuracy With TerraSAR-X: An Update. Geoscience and Remote Sensing Letters, IEEE, 9(5):948-952, Sept 2012.

[7] C. Gisinger, U. Balss, R. Pail, Xiao Xiang Zhu, S. Montazeri, S. Gernhardt, and M. Eineder. Precise Three-Dimensional Stereo Localization of Corner Reflectors and Persistent Scatterers With TerraSAR-X. Geoscience and Remote Sensing, IEEE Transactions on, 53(4):1782-1802, April 2015.

[8] S.M. Kay. Fundamentals of Statistical Signal Processing: Estimation theory. Fundamentals of Statistical Signal Processing. Prentice-Hall PTR, 1998.

[9] R. Bamler and M. Eineder. Accuracy of differential shift estimation by correlation and split-bandwidth interferometry for wideband and delta-k SAR systems. Geoscience and Remote Sensing Letters, IEEE, 2(2):151155, April 2005.

[10] Birgit Wessel, Astrid Gruber, Martin Huber, Markus Breunig, Susanne Wagenbrenner, and Achim Roth. Status of TanDEM-X DEM Calibration and Mosaicking towards the TanDEM-X DEM. In EUSAR 2014; 10th European Conference on Synthetic Aperture Radar; Proceedings of, pages 1-4, June 2014. 